\title{
Fault Diagnosis System for Power Transformers
}

\author{
E. A. Mohamed*, A.Y. Abdelaziz and A. S. Mostafa ** \\ Dept. of Elect. Power \& Machines, Faculty of Eng., Ain Shams \\ University, Cairo, **Ministry of Irrigation, Egypt, and \\ * Qassim Univ., Saudi Arabia
}

\begin{abstract}
This paper introduces an artificial neural network (ANN) based fault diagnosis system (FDS) for power transformers. The system is designed to detect, localize and finally classify faults. The proposed FDS consists of three hierarchical levels. In the first level, a preprocessing procedure for input data is performed. In the second level, an ANN is designed to detect the fault and localize its side. In the third level, there are two sub-diagnosis systems. Each system is dedicated to one side and consists of one ANN designed to classify the fault. This ANN is also cascaded with four parallel ANN's utilized to identify the faulted phase. The performance of FDS is evaluated using samples from local measurements (three-phase primary voltage and primary \& secondary currents). These samples were generated using the EMTP simulation of the High-Dam 15.75/500 kV transformer substation in the $500 \mathrm{kV}$ Upper Egypt network. Different fault types were simulated. Fault location and incipience time were also considered. Evaluation results proved that the performance of the proposed FDS is promising.
\end{abstract}

\section{Introduction}

Fault diagnosis (FD) plays a crucial role in power system monitoring and control that ensures a stable electrical power supply to consumers. FD involves identifying the location and nature of faults occurring on power system due to different disturbances ${ }^{[1,2]}$. FD function is the most basic fault handling function of power system supervisory control and data acquisition (SCADA) systems. Figure 1 shows the different blocks that configure a protective relay, based on which the FDS module is the most important one.

Fault type classification is an essential protective relaying feature due to its significant effect on the enhancement of relaying operation. Correct operation of major protective relays may be depending on fault 
classification [3]. Faulted phase selection is as important as fault detection. It would lead to increasing the system stability and system availability by allowing single pole tripping. Single pole tripping has many benefits like improving the transient stability and reliability of the power system, reducing the switching over-voltages and shaft torsional oscillations of large thermal units ${ }^{[4]}$.

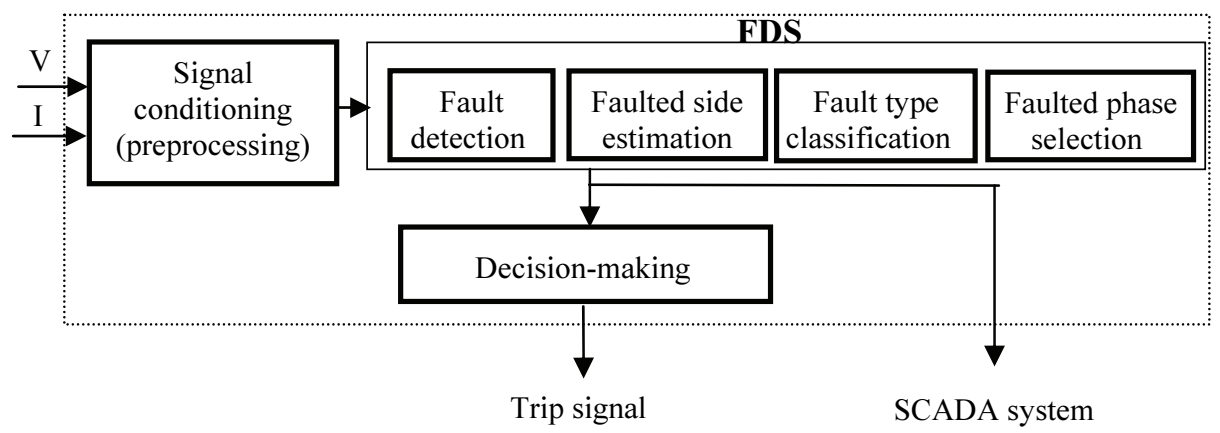

Fig. 1. FDS blocks in a transformer protective relay.

Large power transformers are considered an important equipment in power systems. If a transformer experiences a fault, it is necessary to take it out of service as soon as possible in order to minimize the expected damage. The cost associated with repairing a damaged transformer is very high. An unplanned outage of a power transformer can cost electric utilities millions of dollars. Consequently, it is of great importance to minimize the frequency and duration of unwanted outages of power transformers. Accordingly, high demands are imposed on transformer protective relays. The protection degree of a power transformer is assessed according to its importance and rating [1]. Conventional approaches are: relying on system operating conditions, consuming large time, and failing to perform faulted phase selection ${ }^{[5,6]}$.

ANN's provide a very interesting and valuable alternative because they can deal with most complex situations, which are not defined sufficiently for deterministic algorithms. They are robust with respect to incorrect or missing data. Protective relaying based ANN is not affected by system operating conditions. It also has high computation rates, large input error tolerance and adaptive capability ${ }^{[7]}$. Many literatures concerned with the application of ANN-based protection algorithm for power transformers have been reported ${ }^{[8-11]}$. Most of these literatures are relying on experimental transformer model. However, small size 
transformers usually behave differently compared with large power transformers during inrush and fault periods. Also, the evaluation of ANN relay sensitivity and stability boundaries has not been addressed. In Ref. [12], a transformer model during energization and fault periods is developed using the (EMTP). The training sets for the ANN are formulated considering various conditions including different fault classes. The response of the proposed ANN relay was measured and compared with the differential relay. It was found that the proposed relay was more efficient regarding the speed of detection, sensitivity, and stability boundaries. In this paper, an ANN based FDS, for power transformer external-faults, has been developed. The functions of the proposed FDS are to: detect the fault, localize the faulted side, classify the fault type and determine the faulted phase as well. The required specifications of the FDS should prove high reliability, and fast response.

\section{Power System Simulation Using EMTP}

The EMTP ${ }^{[13]}$ is used for simulating the transients of power system elements including transformers. EMTP transformer model, implemented here, does not handle winding internal faults and inrush current cases, but it gives the ability to adapt the model for the transformers equivalent circuit. The power system considered for this study is the Upper Egypt Power System (UEPS), Fig. 2. It consists of two generating stations, transformer substations, power lines, and loads. The power line from High-Dam $500 \mathrm{kV}$ power station "HD500" to Cairo $500 \mathrm{kV}$ power station "CA500" is, a double circuit line each of length $788 \mathrm{~km}$. The present study is interested in the protection of the High-Dam 15.75/500 kV transformer substation. This system is selected, as an application example, to design and evaluate (test) the proposed approach. Figure (2) shows not only the system arrangement but also the location of the FDS. The UEPS system has been simulated (Appendix A) under the following assumptions:

1- An infinite bus at the CA500 substation was considered.

2- No protection system was activated on the simulated system.

3- Generators were operating at one loading level.

4- No machine controllers were existing.

5- Static loads were modeled as constant impedances. 


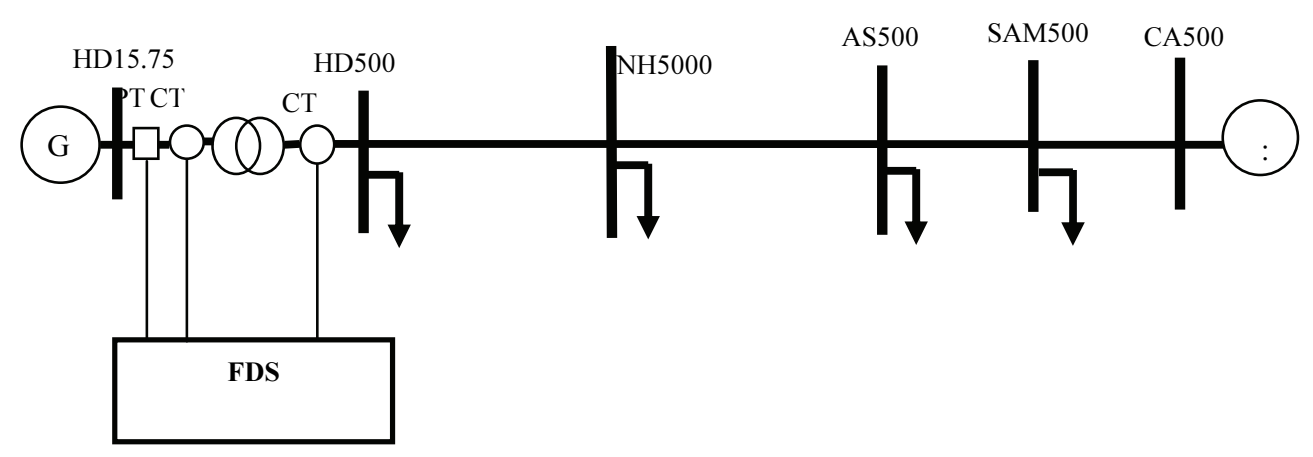

Fig. 2. System under study.

\section{FDS Design Procedure}

\section{FDS Architecture}

The proposed FDS consists of multi ANNs that are arranged to be working in parallel, which make the FDS faster, efficient, robust, accurate and reliable. The suggested FDS consists of three hierarchical levels as illustrated in Fig. 3. The first level is a preprocessing stage involving filtration, conversion, and normalization. In the second level there is one ANN (ANN\#1) which is designed to detect the faulted side. ANN \#1 output has three levels: low level $(<0.3)$ for normal condition, medium level (0.3-0.7 inclusive) for faults on side \#2 (secondary side) and high level $(>0.7)$ for faults on side \#1 (primary side). The third level contains two parallel sub-diagnosis systems (SDS1, SDS2). Each SDS is responsible to classify the fault type and to identify the faulted phase as well. SDS1 is a diagnosis system for side \#1, and consists of one ANN (for fault type classification) in series with four parallel ANNs (for faulted phase selection).

The function of each ANN can be conducted as follows:

a) ANN1P: It is designed to identify the type of fault. Its output has four levels: high levels $(>0.8)$ for L-L-L, high-medium level $(>0.6$ \& $\leq 0.8)$ for $\mathrm{L}-\mathrm{L}-\mathrm{G}$, medium level $(>0.3 \& \leq 0.6)$ for $\mathrm{L}-\mathrm{L}$, and low level $(\leq 0.3)$ for $\mathrm{L}-\mathrm{G}$ faults.

b) ANN11: This ANN is to identify the phase to ground faults. The network is activated by ANN1P. The network output has three levels, 
high level $(\geq 0.7)$ for R-G, medium level $(>0.3 \&<0.7)$ for $\mathrm{S}-\mathrm{G}$, and low level $(\leq 0.3)$ for $\mathrm{T}-\mathrm{G}$ faults.

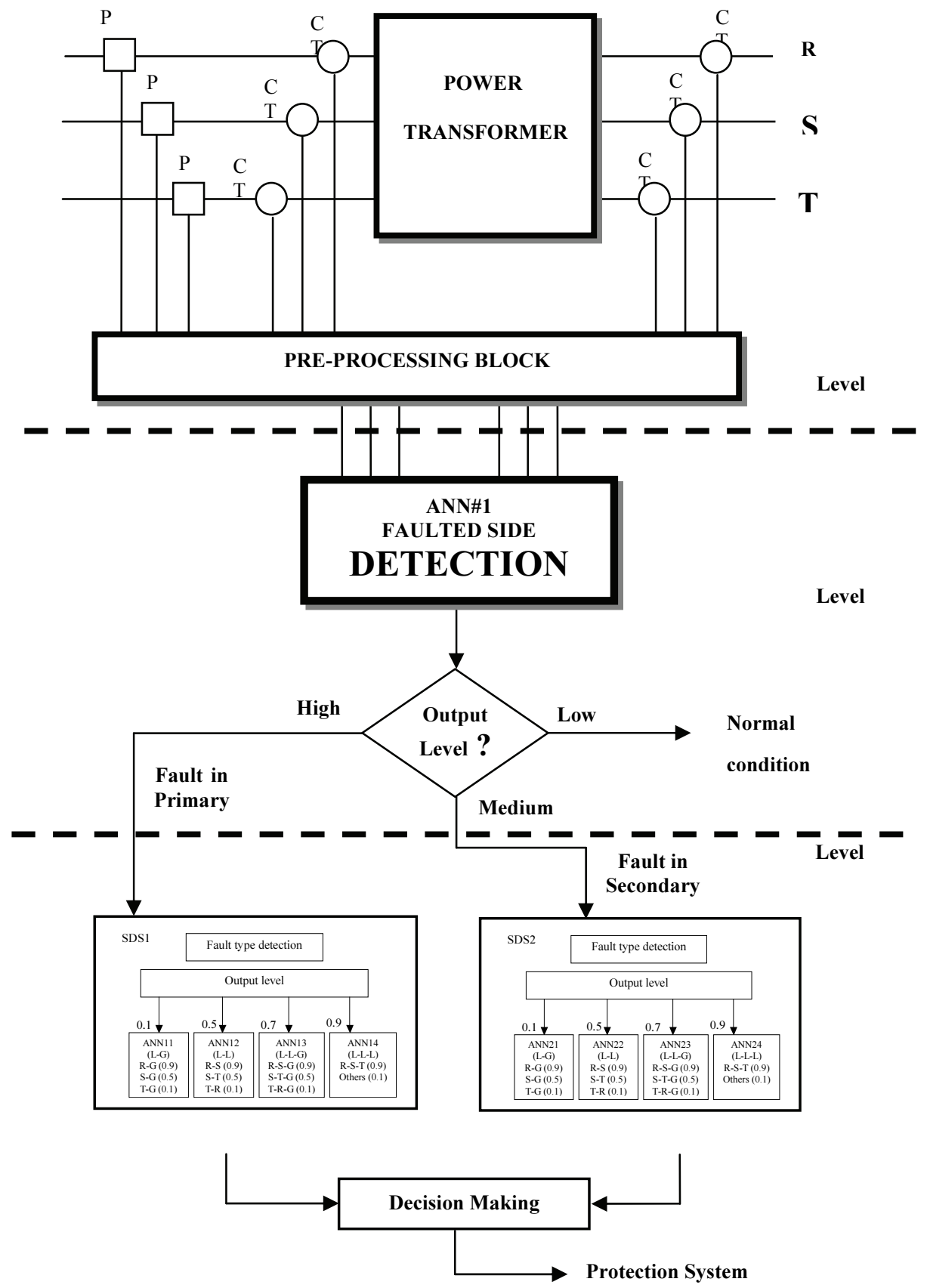

Fig. 3. Architecture of Suggested FDS. 
c) ANN12: This ANN is to classify the phase to phase faults. It is triggered by the output of ANN1P. Its output has three levels, high level $(\geq 0.7)$ for R-S, medium level $(>0.3 \&<0.7)$ for $\mathrm{S}-\mathrm{T}$, and low level $(\leq 0.3)$ for T-R faults.

d) ANN13: This ANN is to recognize the double phase to ground faults. It is activated by the output of ANN1P. The output is high levels $(\geq 0.7)$ for R-S-G, medium level $(>0.3 \&<0.7)$ for $\mathrm{S}-\mathrm{T}-\mathrm{G}$, and low level $(\leq 0.3)$ for T-R-G faults.

e) ANN14: This ANN is to identify three phase to ground faults. The network gives high level $(>0.5)$ output for three phase to ground faults and $(\leq 0.5)$ for else.

Similarly, SDS2 is the sub-diagnosis system for faults on side \# 2 . It consists of ANN1S for fault type classification and (ANN21, ANN22, ANN23 and ANN24) for phase selection.

\subsection{ANN Design}

The error back-propagation technique with adaptive learning rate and momentum is used (Appendix B). The three-layer feed-forward ANN is satisfactory for most power applications ${ }^{[14]}$. The tan-sigmoid and log-sigmoid functions are differentiable as well as monotonic functions. Therefore, selected design will have one hidden layer with tan-sigmoid neurons followed by an output layer with log-sigmoid neurons.

Since, the proposed FDS would determine the faulted side, fault type and faulted phase, it was decided to choose three phase voltage and current samples as input signals. This selection is based on the fact that the three-phase current and voltage capture all the required information about the fault (as side, type and incipient time). A sampling rate of 800 $\mathrm{Hz}$ (16 samples per cycle for $50 \mathrm{~Hz}$ power frequency) is used. This sampling rate satisfies the feature space requirements and gives optimal conversion performance ${ }^{[14]}$. On the other hand, one output neuron with multi-level is used in this research.

There are 11 ANN's constructing the proposed FDS, therefore, it will be a tedious work to design each ANN individually. One ANN is, therefore, selected as model. After selecting the number of inputs and \# of neurons in the hidden layer for the model, these selections were then applied to the other ten. Therefore, the input to each of these ANN's may 
contain one or more samples. The set of samples which are used as inputs to the ANN will be called a pattern. A sample means a vector of three phase primary voltages and primary \& secondary currents, sampled at a certain instant. Thus the number of inputs of a certain pattern is defined as follows:

Number of input patterns $=9 \times$ number of samples composing the pattern $=9 \times 4=36$. Different configurations of ANN's were tested. It was found that, ANN with 36 neurons in one hidden layer have resulted in a good performance.

\subsection{Design and Evaluation Samples}

The system under study is subjected to different types of faults at the primary and secondary sides of the High-Dam transformer substation. Three phase primary voltages, primary and secondary currents have been calculated corresponding to different fault types, fault side and/or fault incipience time. Figure 4 presents a sample of these results. Ten fault types (R-G, S-G, T-G, R-S, S-T, T-R, RS-G, ST-G, TR-G, RST-G) are simulated. Two different fault locations (primary and secondary sides) as well as three different fault incipience times (0, 45, 90 degrees) were considered.

Number of fault cases $=10$ fault types $\times 2$ fault location sides $\times 3$ incipient times $=60$ cases

Therefore, sixty case studies were performed. Each case study contains 5.5-cycles during the fault and 7.5-cycles after clearance. In addition, two-cycles for pre-fault conditions were taken into consideration. Therefore, the number of samples is 14400 samples $(60 \mathrm{x}$ 240). The generated samples were divided into two sets; the design set which is composed of 9600 samples (40 case x 240 samples) and the evaluation set which is composed of 4800 samples (20 case x 240 samples).

It can be summarized that the design cases (40) include 4 fault types (L-G, L-L, L-L-G, L-L-L), 2 fault locations (primary or secondary) and 2 fault incipient times $\left(0,90^{\circ}\right)$, while the evaluation cases (20) include 4 fault types, 2 fault locations and one fault incipient time $\left(45^{\circ}\right)$. All case studies generated using the EMTP, are then loaded into MATLAB. The voltages and currents are normalized and reshaped in the form of group of patterns, where each pattern is composed of four 
consecutive samples. The ANN-Toolbox of MATLAB ${ }^{[15]}$ is used to design each ANN. Figure 5 presents the structure of each $\mathrm{ANN}^{[16]}$.

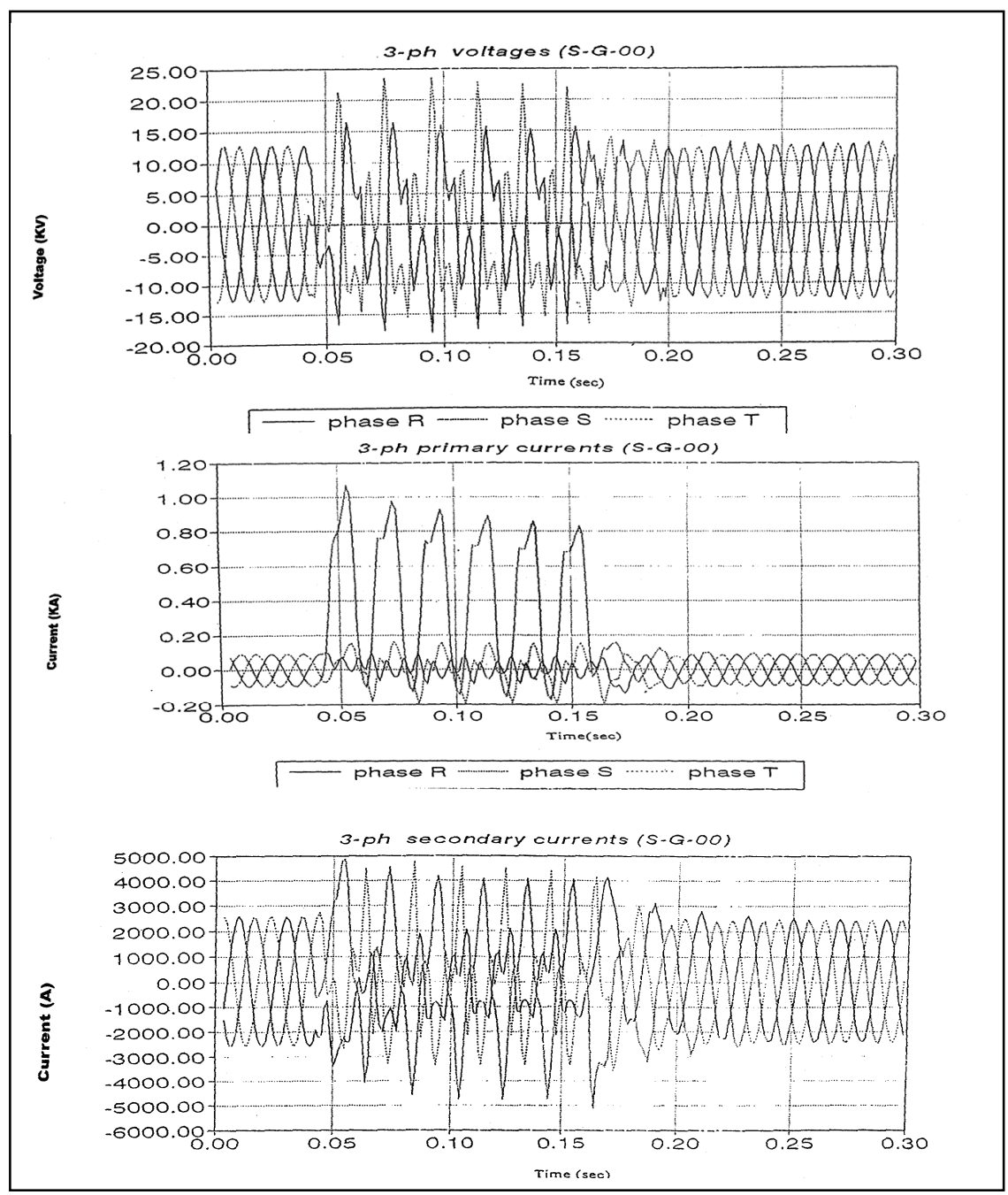

Fig. 4. Primary Voltage, Primary $\left(\mathbf{x 1 0}^{3}\right)$ \& Secondary Currents for L-G Fault.

\section{FDS Evaluation Results}

\subsection{Faulted Side Detection (ANN\#1)}

ANN\#1 is designed to detect the occurrence of fault and to locate its side, primary or secondary. Figure 6 shows a sample of the output evaluation results ( 8 cases only are included). Each case study includes pre-fault, during 
fault, and post-fault periods. Pre-fault as well as post-fault conditions are indicated as normal (0.1 level), while during fault is indicated as primary fault ( 0.9 level) or secondary fault $(0.5$ level). Dashed line is the predicted output while solid line is the target output. It can be seen that the network is capable for the detection of the faulted side.

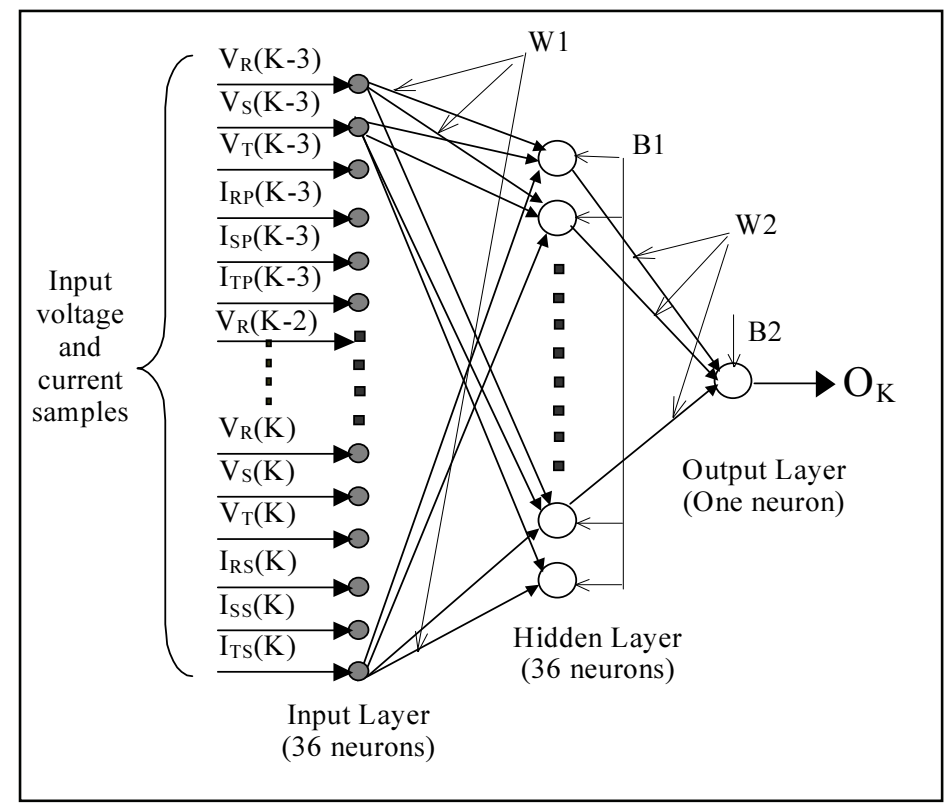

Fig. 5. Three Layers ANN structure.

\subsection{Fault Type Classification}

ANN1P: It is designed to discriminate between different fault types located on the primary side. There are four output levels for this network. The output levels $0.1,0.5,0.7 \& 0.9$ represent L-G, L-L, L-L-G and L-L-L faults, respectively. Sample of training results are included in Appendix B. Figure 7 shows the obtained sample of evaluation results. It is clear to prove that different fault types were correctly classified using this ANN.

ANN1S: Similarly, it is designed (sample of training results are shown in Appendix B) to identify between different fault types located on the secondary side. Figure 8 shows the obtained results of a sample of the evaluation cases. It can be seen that the classification performance of ANN1S is accurate. 


\subsection{Faulted Phase Estimation}

$\boldsymbol{L}-\boldsymbol{G}$ faults: In this case ANN11 \& ANN21 are designed. Each network is designed to detect L-G faults on one side. For example, ANN11 is used to detect and to identify the faulted phase on the primary side. There are $18 \mathrm{~L}-\mathrm{G}$ fault cases for design and 9 evaluating cases on each side. An output sample of evaluation results are shown in Fig. 9 \& 10 , respectively. The output levels of $0.9,0.5 \& 0.1$ indicate $\mathrm{R}-\mathrm{G}, \mathrm{S}-\mathrm{G}$ and T-G faults, respectively. It can be concluded that the classification performance of the two networks is accurate.
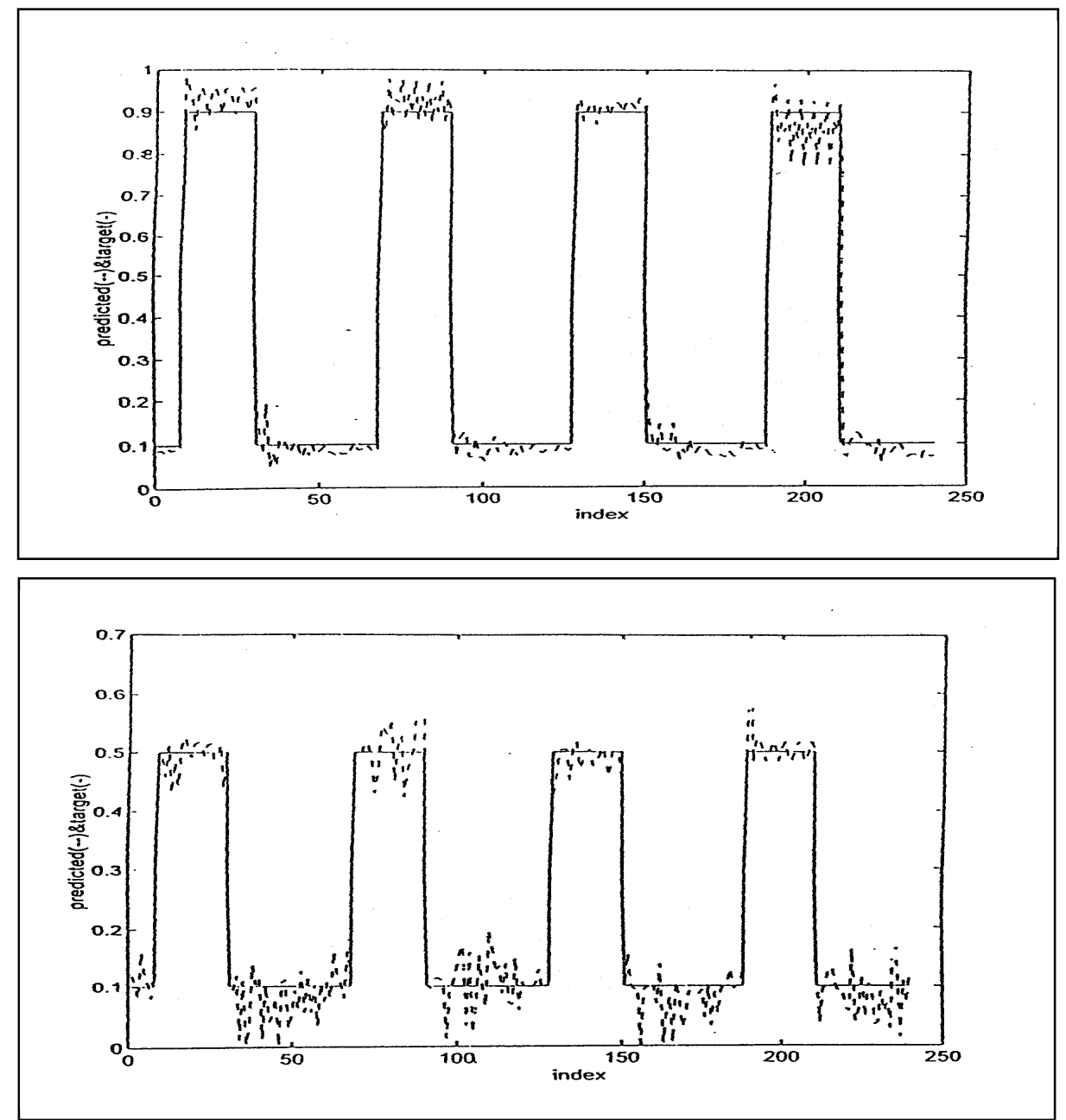

Fig. 6. ANN\#1, evaluating sample results.

(0.1) normal, $(0.5)$ secondary faults and $(0.9)$ primary faults 

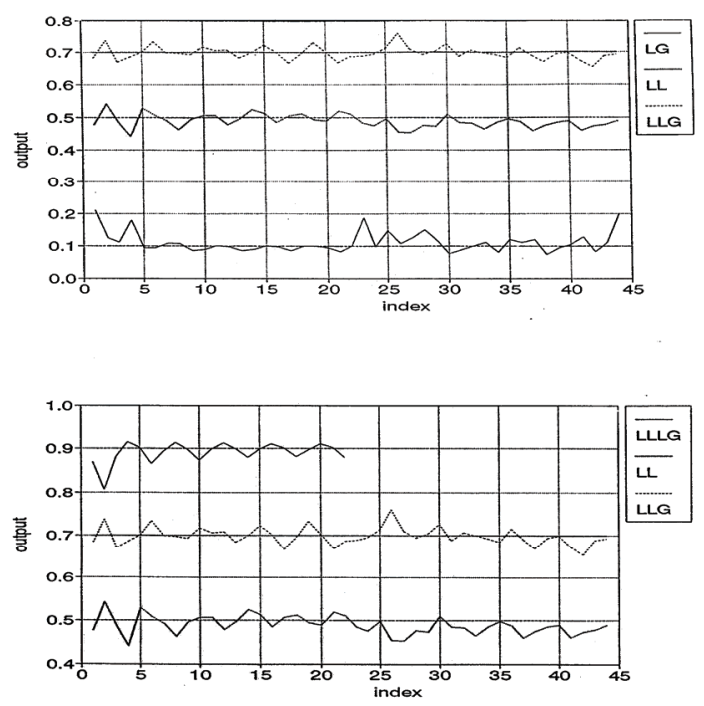

Fig. 7. ANN1P, evaluating sample results.

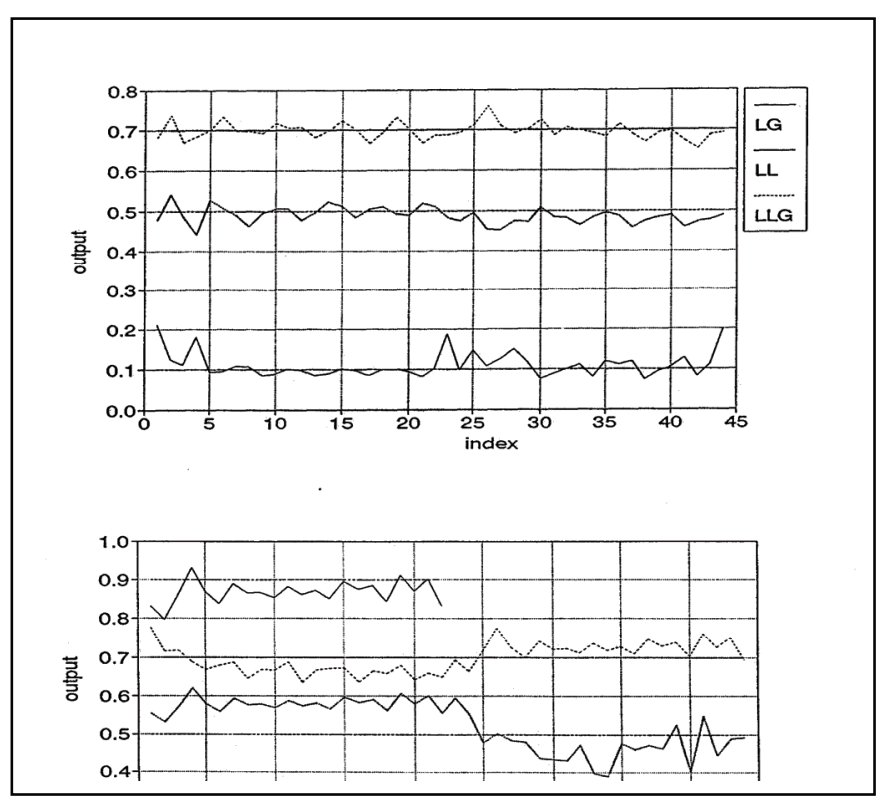

Fig. 8. ANN1S, evaluating sample results. (0.1) L-G, (0.5) L-L, (0.7) L-L-G and (0.9) L-L-L 

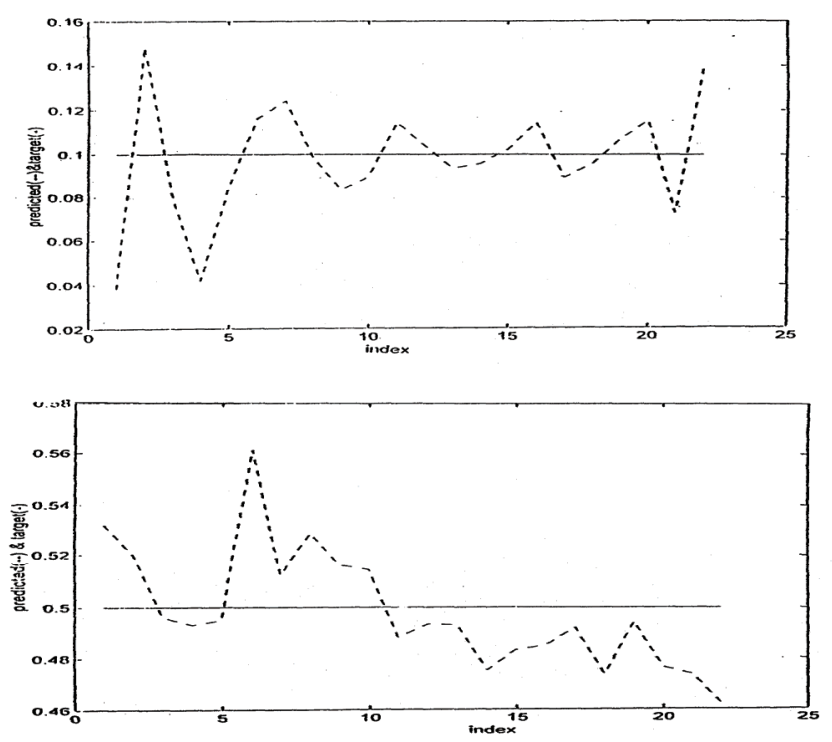

Fig. 9. ANN11, evaluating sample results.

(0.1) T-G \& (0.5) S-G
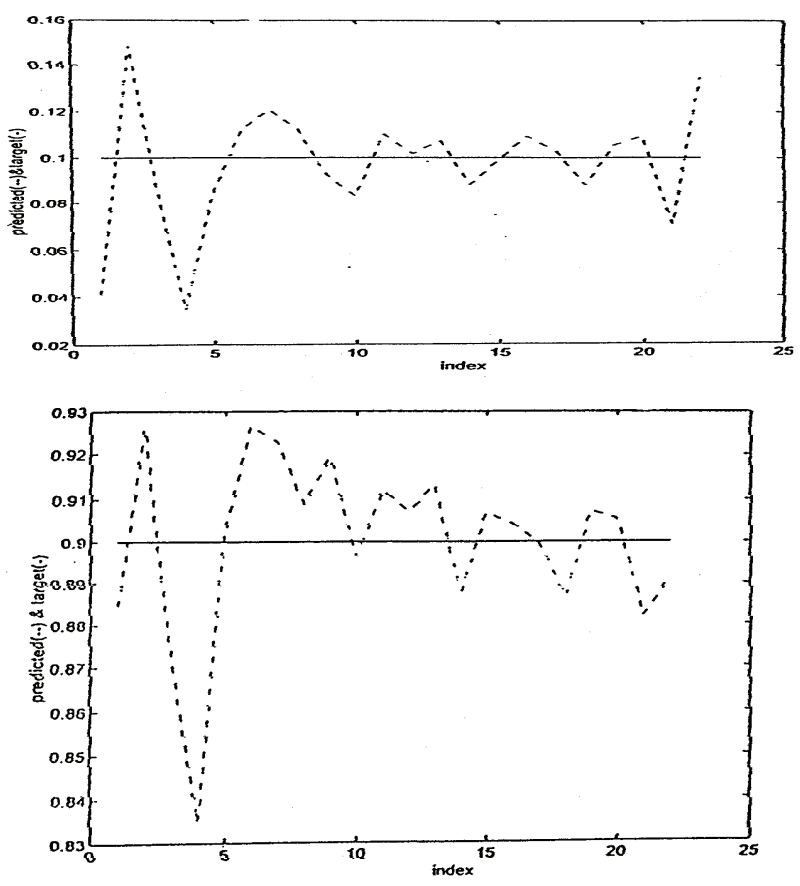

Fig. 10. ANN21, evaluating sample results

(0.1) T-G \& (0.9) R-G 
L-L faults: For this type of fault ANN12 \& ANN22 are designed. Figures 11 and 12 show an output sample of the evaluation results. The output levels of $0.9,0.5 \& 0.1$ indicate R-S, S-T and T-R faults, respectively. It can be stated that L-L faults can be accurately classified.
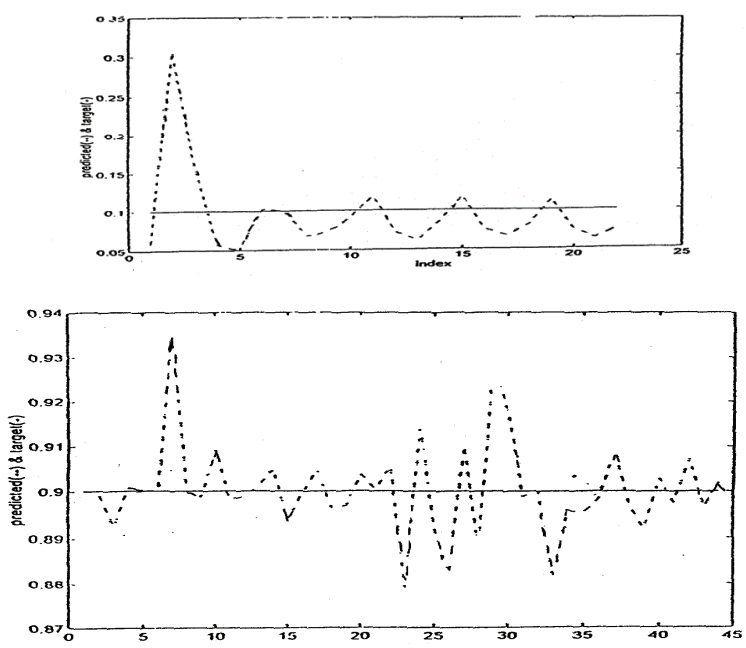

Fig. 11. ANN12, evaluating sample results.

(0.1)T-R \& (0.9) R-S
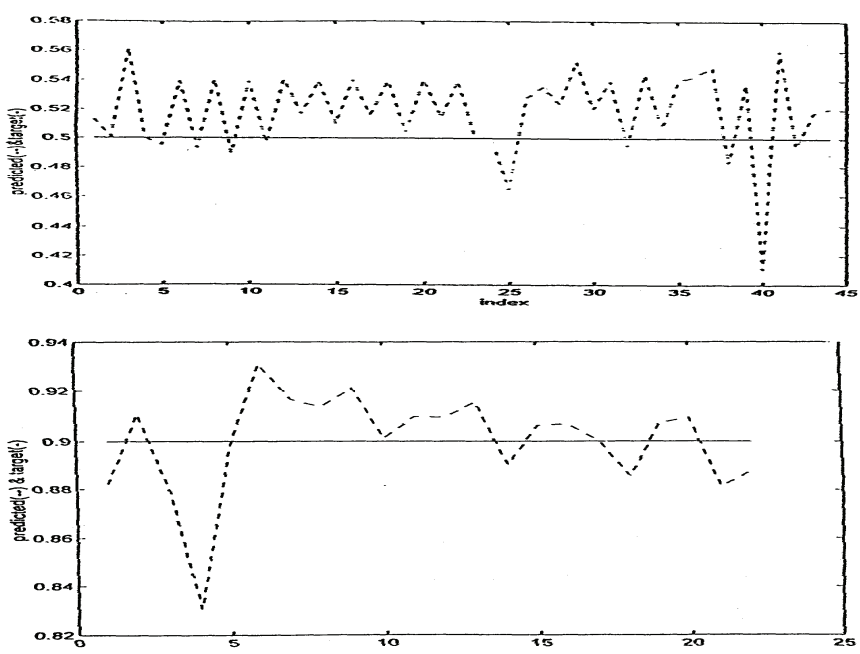

Fig. 12. ANN22, evaluating sample results.

(0.5) S-T \& (0.9) R-S 
L-L-G faults: ANN13 \& ANN23 are designed and then evaluated in this case. Figures 13 and 14 present an output sample of the evaluation results. The output levels $0.9,0.5 \& 0.1$ indicate R-S-G, S-T-G and T-R$\mathrm{G}$ faults, respectively. The response of each network is found satisfactory.
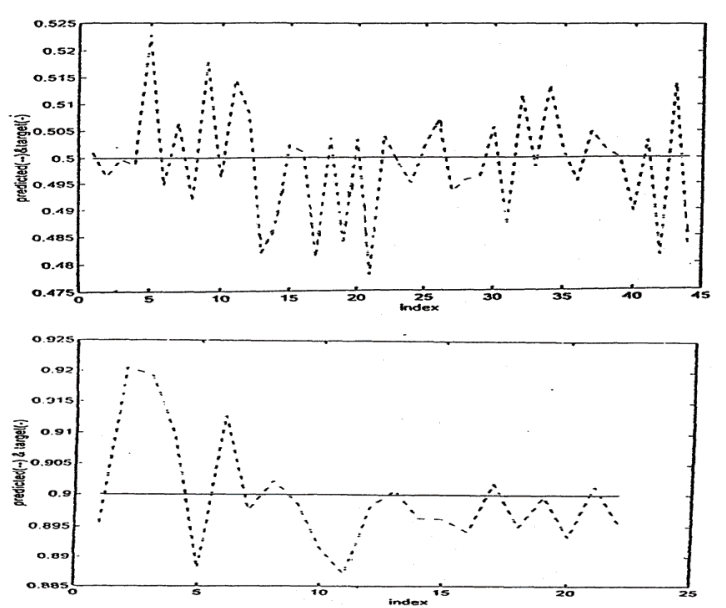

Fig. 13. ANN13, evaluating sample results. (0.5) S-T-G \& (0.9) R-S-G
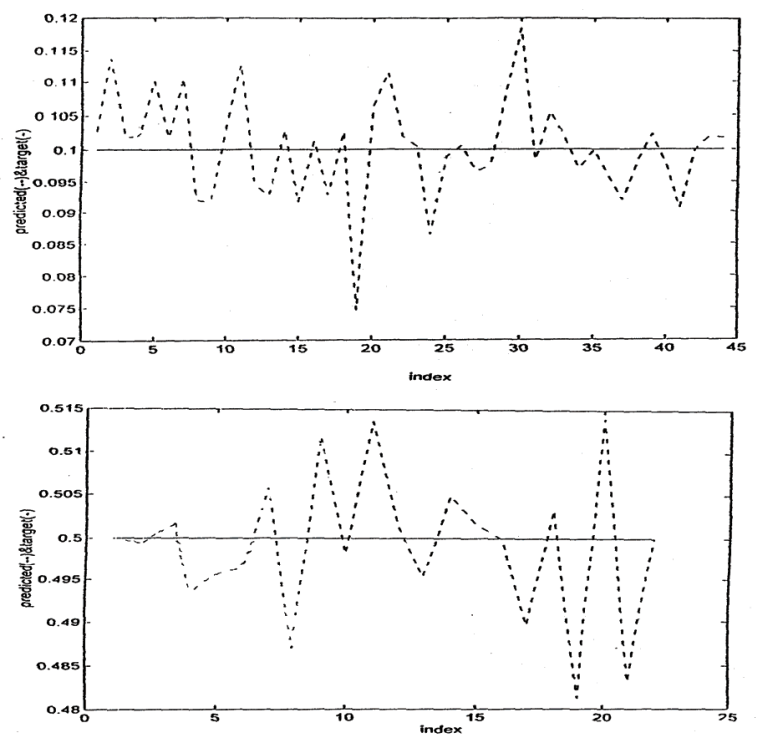

Fig. 14. ANN23, evaluating sample results. (0.1) T-R-G \& (0.5) S-T-G 
$\boldsymbol{L}-\boldsymbol{L}-\boldsymbol{L}-\boldsymbol{G}$ faults: Networks ANN14 and ANN24 are designed for this fault type. Figures 15 and 16 present an output sample of evaluating results. Based on these results, these networks have achieved an acceptable performance.

From the previous results, it can be stated that fault detection, faulted side location and fault classification were performed accurately using the proposed FDS.

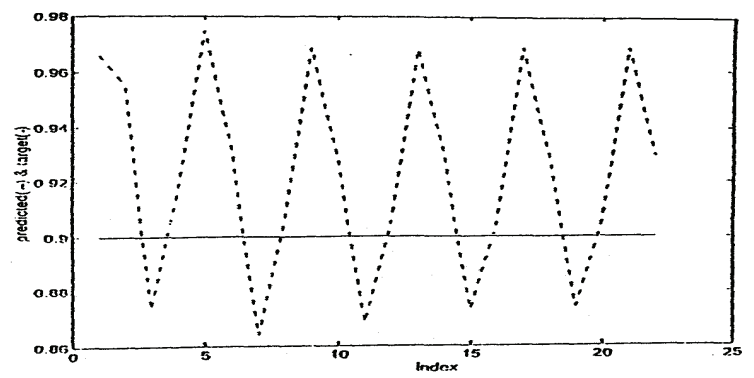

Fig. 15. ANN14, evaluating sample results.

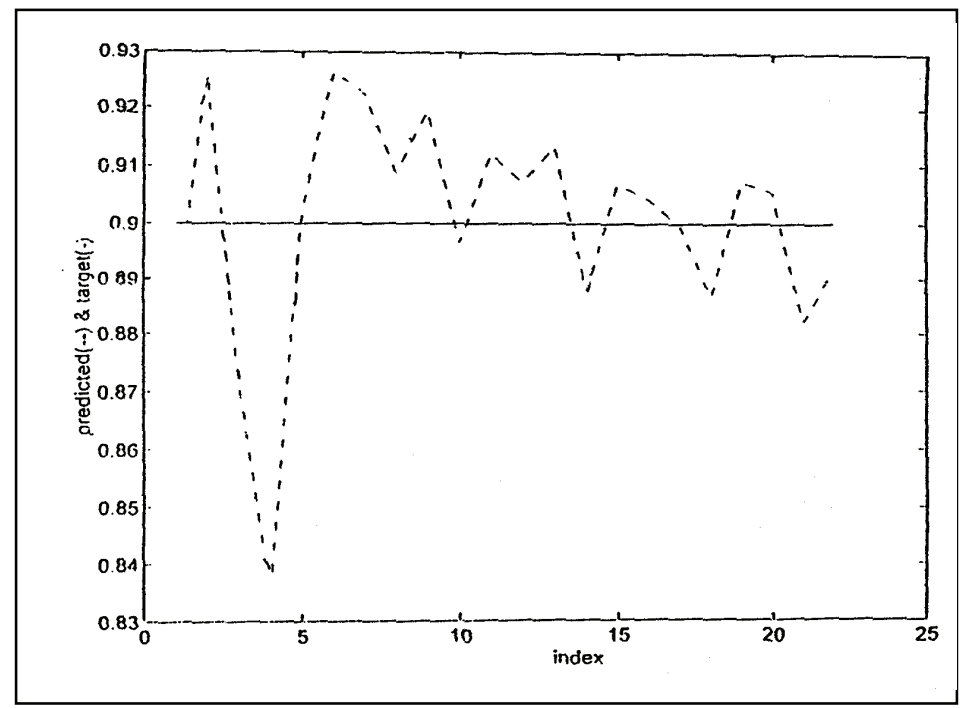

Fig. 16. ANN24, evaluating sample results.

(0.9) R-S-T, and 0.1 others 


\section{Conclusions}

The design details of the ANN-based fault diagnosis system (FDS) for power transformers, specially for external faults, have been presented. The evaluation of the performance of the proposed FDS under various fault conditions leads to the following conclusions: 1- The proposed architecture of the proposed FDS has the advantage of assigning one task to each ANN; 2- The adequate length of the data window is $1 / 4$ cycle (4 samples of: three phase voltages, primary and secondary currents); 3The time response of the proposed FDS is fairly fast due to its parallel structure; 4-The selected design of each ANN is suitable (36 input nodes, 36 hidden-neurons and one output-neuron); 5- The FDS performance in the evaluation phase was accurate, irrespective of fault location, fault type, and fault incipience time; 6- The FDS can be used as a part of a new generation of very high speed power transformer protective relays.

\section{References}

[1] Kasztenny, B. and Kezunovic, M., "Digital Relays Improve Protection of Large Transformers", IEEE Computer Applications in Power, October 1998, pp: 39-45.

[2] Sekine, Y. et al., "Fault Diagnosis of Power Systems", Proceedings of the IEEE, 80(5): 673683, May (1992).

[3] Westinghouse Electric Corporation, Applied Protective Relaying, Relay Investment Division, Coral Springs, Florida 33065(1982).

[4] IEEE working group- power system relaying committee, "Single Phase Tripping and Autoreclosing of Transmission Lines", IEEE Trans. on Power Delivery, 7(1) Jan. (1992).

[5] Dalsten, T. and Friedrick, T., "Multi-Neural Network Based Fault Area Estimation for High Speed Protective Relaying", IEEE Trans. on Power Delivery, 11 ( 2 ) April (1996) .

[6] Kezunovic, M. and Rikalo, I., "Detect and Classify Faults Using Neural Nets", IEEE Transactions on Computer Applications in Power, 9(4) October (1996).

[7] CIGRE TF 38.06.06, "Artificial Neural Networks for Power Systems", Electra, No. 159:77101, April (1995).

[8] Lui, P. and Malik, O.P. , "Improved Operation of Differential Protection of Power Transformers for Internal Faults", IEEE Trans. on Power Delivery, 7(4): 1912-1919 (1992).

[9] Yabe, Y., "Power Differential Method for Discrimination Between Fault and Magnetizing Inrush Current in Transformer", IEEE Trans.on Power Delivery, 12(3): 1109-1118 (1997).

[10] Zaman, M.R. and Rahman, M.A., "Experimental Testing of an Artificial Neural Network Based Protection of Power Transformer", IEEE Trans. on Power Delivery, 13(2): 510-517 (1998).

[11] Perez, L.G. et al., "Training an Artificial Neural Network to Discriminate Between Magnetizing Inrush and Internal Faults", IEEE Trans. on Power Delivery, 9(1): 434-441 (1994). 
[12] Darwish, H.A. and Elnabawy, E., "An Artificial Neural Network-Based Relay for Power Transformer Protection', Proceedings of MEPCON'2000, Ain Shams University, Cairo, pp: $595-600$.

[13] EMTP Developed Coordination Group; Electric Power Research Institute EMTP Rule Book, Version 2.1, Sections 6-10 (1993).

[14] El-Sharkawy, M. A., "Overview of Neural Network Application to Power Systems", Proceedings of 5-th, MEPCON'97, Alexandria, Egypt, Jan.4-6 (1997).

[15] Demuth, H. and Beal, M., Neural Network - Toolbox for Use with Matlab (1992).

[16] Mohamed, E.A., Abdelaziz, A.Y. and Mustafa, A.S., "On-Line Fault Diagnosis of Power Transformers", Proceedings of 10-th Int. Middle East Power Conference (MEPCON), Dec.1315, 2005, Port-Said, Egypt, pp: 207-15. 


\section{Appendix A \\ Power System Simulation Using EMTP}

\section{General}

The EMTP is used for simulating the transients in power system elements including transformers. EMTP transformer model can not directly give winding internal fault and inrush current cases but it gives the ability to adapt the model for the transformers equivalent circuit. The power system considered here for this study is the Upper Egypt Power System (UEPS). It consists of generating stations, substation transformers, power lines, and loads. The power line, starting from the High-Dam 500 $\mathrm{kV}$ "HD500" generating station and ending at Cairo $500 \mathrm{kV}$ "CA500" substation, is a double circuit line each of length $788 \mathrm{~km}$, considering HD500 as a sending end and CA500 station as a receiving end. The line passes through Nagh-Hammady (NH500) at distance $235 \mathrm{~km}$ from the High-Dam then it reaches Samalut (SA500) at distance of 343 $\mathrm{km}$ from NH500 and $209 \mathrm{~km}$ from CA500. The main transformer substation considered in this study is the High-Dam generating-station substation, it consists of 12 units, each of $15.75 / 500 \mathrm{kV}, 206$ MVA capacity. On the other hand, the High-Dam generatingstation itself contains also twelve generators each of $175 \mathrm{MW}$ capacity .

\section{EMTP Digital Simulation}

The Electro-magnetic transient program (EMTP) is a well known world-wide program. It is used to solve the equations describing the system under transient conditions. This program consists of a number of functional modules, which separately contains mathematical models of various components. The major functional modules are transformers, transmission lines, switches, surge arresters, control systems, and electric machinery.

The synchronous machine is simulated using the EMTP developing module. The transient model is employed in this study. All transmission lines (overhead transmission lines and underground cables) are represented by their $\pi$ equivalent network with their series R-L and shunt G-C lumped parameters as a suitable representation for the phenomenon under study. All loads are simulated by their equivalent R-L-C parameters as constant impedances. Each three-phase transformer is simulated by three singlephase transformers, each of them is represented by its transient model. The EMTP can be used to represent an infinite bus with a constant voltage, and constant frequency. Figure 1 presents a sample of simulation results.

\section{System Data}

The system data and the operating conditions are given as follows:

\section{Generator Data:}

Where:

\begin{tabular}{|c|c|c|c|c|c|c|c|c|}
\hline $\mathbf{X}_{\mathbf{d}}{ }^{\prime}$ & $\mathbf{X}_{\mathbf{d}}{ }^{\prime \prime}$ & $\mathbf{X}_{\mathbf{d}}$ & $\mathbf{X}_{\mathbf{q}}{ }^{\prime}$ & $\mathbf{X}_{\mathbf{q}}{ }^{\prime \prime}$ & $\mathbf{X}_{\mathbf{q}}$ & $\boldsymbol{\tau}_{\mathbf{d}}{ }^{\prime}$ & $\mathbf{H}$ & $\mathbf{D}$ \\
\hline 0.4 & 0.37 & 1.2 & 0.36 & 0.16 & 0.79 & 2.8 & 6 & 0.18 \\
\hline
\end{tabular}

$\mathbf{X}_{\mathrm{d}}{ }_{\mathrm{d}}^{\prime \prime}, \mathbf{X}_{\mathbf{d}}{ }^{\prime} \& \mathbf{X}_{\mathrm{d}}$ : d-axis subtransient, transient \& synchronous reactance's respectively;

$\mathbf{X}_{\mathbf{q}}{ }^{\prime \prime}, \mathbf{X}_{\mathbf{q}} \backslash \& \mathbf{X}_{\mathbf{q}}$ : q-axis subtransient, transient \& synchronous reactance's respectively; 
$\tau_{\mathbf{d}}{ }^{\prime}: \mathrm{d}$-axis transient time constant (sec); $\mathbf{H}$ : inertia time constant (sec); $\mathbf{D}:$ damping factor.

Transformer Data: $206 \mathrm{MVA}, 15.75 \Delta / 500 \mathrm{Y} \mathrm{kV}, \mathrm{X}(\mathrm{LV})=0.006 \Omega, \mathrm{X}(\mathrm{HV})=1.67 \Omega$ Transmission Lines Data
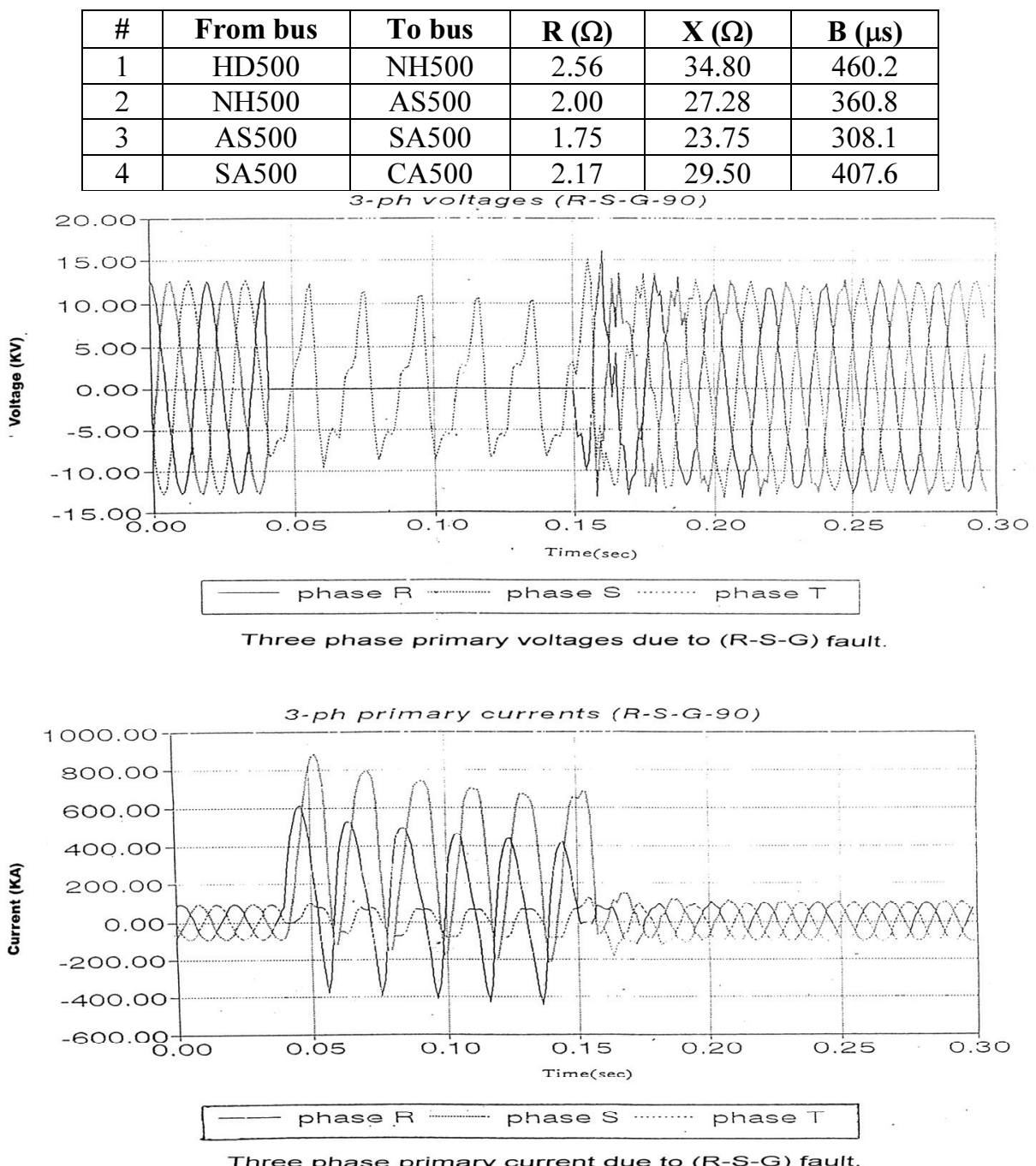

Fig. 1. Three-phase primary voltages, primary currents, secondary currents due to $L$ (phase R)-L (phase S)-G primary fault at $90 \mathrm{deg}$ incipient angle(90). 


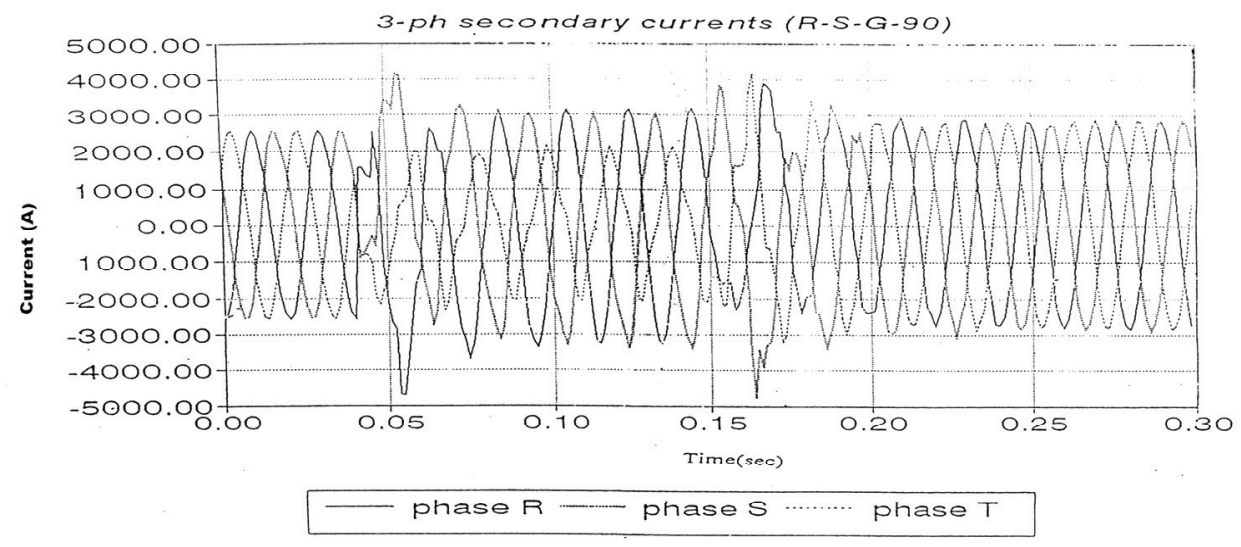

Three phase secondary current due to (R-S-G) fault

Fig. 1. Contd.

Operating Conditions:

\begin{tabular}{|c|c|c|}
\hline Bus & $\mathbf{V ~ ( k V )}$ & Load (MVA) \\
\hline HD500 & $523.38 \angle 22.9$ & $10.32+\mathrm{j} 103.32$ \\
\hline NH500 & $507.70 \angle 11.8$ & $198.30+\mathrm{j} 132.17$ \\
\hline AS500 & $505.34 \angle 6.5$ & $85.12+\mathrm{j} 42.56$ \\
\hline SA500 & $496.70 \angle 3.2$ & $45.44+\mathrm{j} 40.89$ \\
\hline CA500 & $475.00 \angle 0$ & $157.43+\mathrm{j} 107.6$ \\
\hline
\end{tabular}




\section{Appendix B \\ ANN Design Algorithm}

\section{Architecture of ANN}

An artificial neural network can be defined as a parallel distributed information processing structure consists of processing elements (neurons) interconnected together with unidirectional signal channels called connections. ANN consists of input, an output and possibly many hidden layers. The input layer is not a neural computing layer because the nodes in this layer have no input weights or activation function so they called passive nodes. But the hidden and output layers consist of neurons (active nodes). The way the neurons are connected in the ANN layer is specific for the different existing models.

\section{Artificial Neuron}

An artificial neuron is the basic building block of ANN. It acts as a simplified processing unit. Figure 2 shows the schematic representation of an artificial neuron. The input signals are $X_{1}, X_{2}, \ldots, X_{n}$. Each neuron's input is weighted with an appropriate $w$. The sum of weighted inputs along with the bias offset are input to the activation function F. Neuron may use any differentiable function to generate their output.

$$
o_{\mathrm{j}}=F_{j}\left(\mathrm{X}+b_{j}\right) \quad \& \quad \mathrm{X}=\sum_{i=1}^{n} w_{i j} * I_{i}
$$

where:

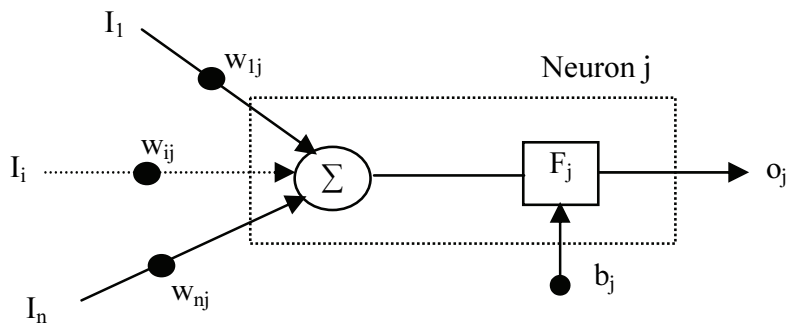

Fig. 2. An illustration of artificial neuron.

$\mathrm{I}_{\mathrm{i}} \quad: \quad \mathrm{i}^{\text {th }}$ input to neuron.

$\mathrm{w}_{\mathrm{ij}}$ : Connection weight between input $\mathrm{i}$ and neuron $\mathrm{j}$.

$b_{j} \quad$ : The bias value of neuron $j$.

$F_{j}$ : The activation function of neuron $j$.

$\mathrm{o}_{\mathrm{j}} \quad$ : The output of neuron $\mathrm{j}$.

\section{Tan-Sigmoid Activation Function}

This function is used to draw the neuron output in the interval of -1 and +1 , as the input varies from plus and minus infinity. This function is fully differentiable and its mathematical model is as follows: $F(x)=\frac{1-e^{-2 x}}{1+\mathrm{e}^{-2 x}}$ 


\section{Design Procedure for ANN Using the Back-Propagation Algorithm}

The back-propagation learning rules are used to adjust the weights and biases of networks so as to minimize the sum squared error of network. This is done by continually changing the values of the network weights and biases in the direction of steepest descent with respect to error. This is called a gradient descent procedure. Changes in each weight and bias are proportional to that element's effect on the sumsquared error of the network. Typically, a new input will lead to an output similar to the correct output for input vectors used in training that are similar to the new input being presented. This generalization property makes it possible to train a network on a representative set of input/target pairs and get good results for new inputs without training the network on all possible input/output pairs. A summary of steps for the backpropagation training technique may be as follows:

1- Initialize the network weights and biases by small random elements.

2- Present a data pattern from the training set with input and desired output/target pairs.

3- The actual outputs will be calculated by the sigmoid neurons. minimized.

4- Calculate the error, the difference between target and actual output, to be

5- Derivatives of error (called delta vectors) are calculated for the output layer and then back propagated through the network until delta vectors are available to the hidden layers. For the sigmoid transfer function

$$
\begin{aligned}
& \delta_{j}=\left(t_{j}-o_{j}\right) o_{j}\left(1-o_{j}\right) \quad \text { for node } \mathrm{j} \text { on the output layer } \\
& \delta_{j}=o_{j}\left(1-o_{j}\right) \sum_{i} w_{i j} \delta_{j} \quad \text { for node } \mathrm{j} \text { on the hidden layer }
\end{aligned}
$$

6- The weights and biases changes are calculated recursively backwards from the output layer towards the input layer. Once all changes are calculated the weights and biases are updated as follows:

$$
\begin{gathered}
w_{j i}(k+1)=w_{j i}(k)+\Delta w_{j i}(k) \\
b_{j}(k+1)=b_{j}(k)+\Delta b_{j}(k) \\
\Delta w_{j i}(k)=\eta \delta_{j} O_{i} \& \Delta b_{j}(k)=\eta \delta_{j}
\end{gathered}
$$

where:

$\Delta w_{j \mathrm{i}}$ : weight change from a node in $\mathrm{i}^{\text {th }}$ layer to a node in $\mathrm{j}^{\text {th }}$ layer.

$w_{j i}$ : weight from layer i to layer $\mathrm{j}$. \& $b_{j}$ : the baises for the layer $\mathrm{j}$.

$\delta_{j}$ : delta vector of $\mathrm{j}^{\text {th }}$ layer $\& o_{j}$ : the output of network $\mathrm{j}^{\text {th }}$ layer.

$o_{i}:$ the input to network $\mathrm{j}^{\text {th }}$ layer. $\& \eta$ : Learning rate

$k$ : iteration number \& $t$ : target vector

7- A method called momentum is used, it allows a network respond not only to the local gradient but also to recent trends in the error surface. When the momentum constant $(\alpha)$ is 0 a weight change is based slowly on the gradient, but when it is 1 the new weight change is set to equal the last weight change. Back propagation with momentum is expressed mathematically as: 


$$
w_{j i}(k+1)=w_{j i}(k)+\eta \delta_{j} o_{i}+\alpha \Delta w_{j i}(k),
$$

8- Using an adaptive learning rate, that attempts to keep the learning step as large as possible, leads to reduce the training time.

9- Initial conditions for two-layer network can be chosen more favorably than by using purely random numbers. If you can pick more favorable initial conditions the learning rule does not need to work as hard and training time is reduced.

10- Present the next pattern in the training set and repeat steps from 1 to 9.

11- Check the error goal or a predefined maximum number of iterations. Steps from 1 to 10 will be repeated until one of them is achieved. (See program flow chart in Fig. 3).

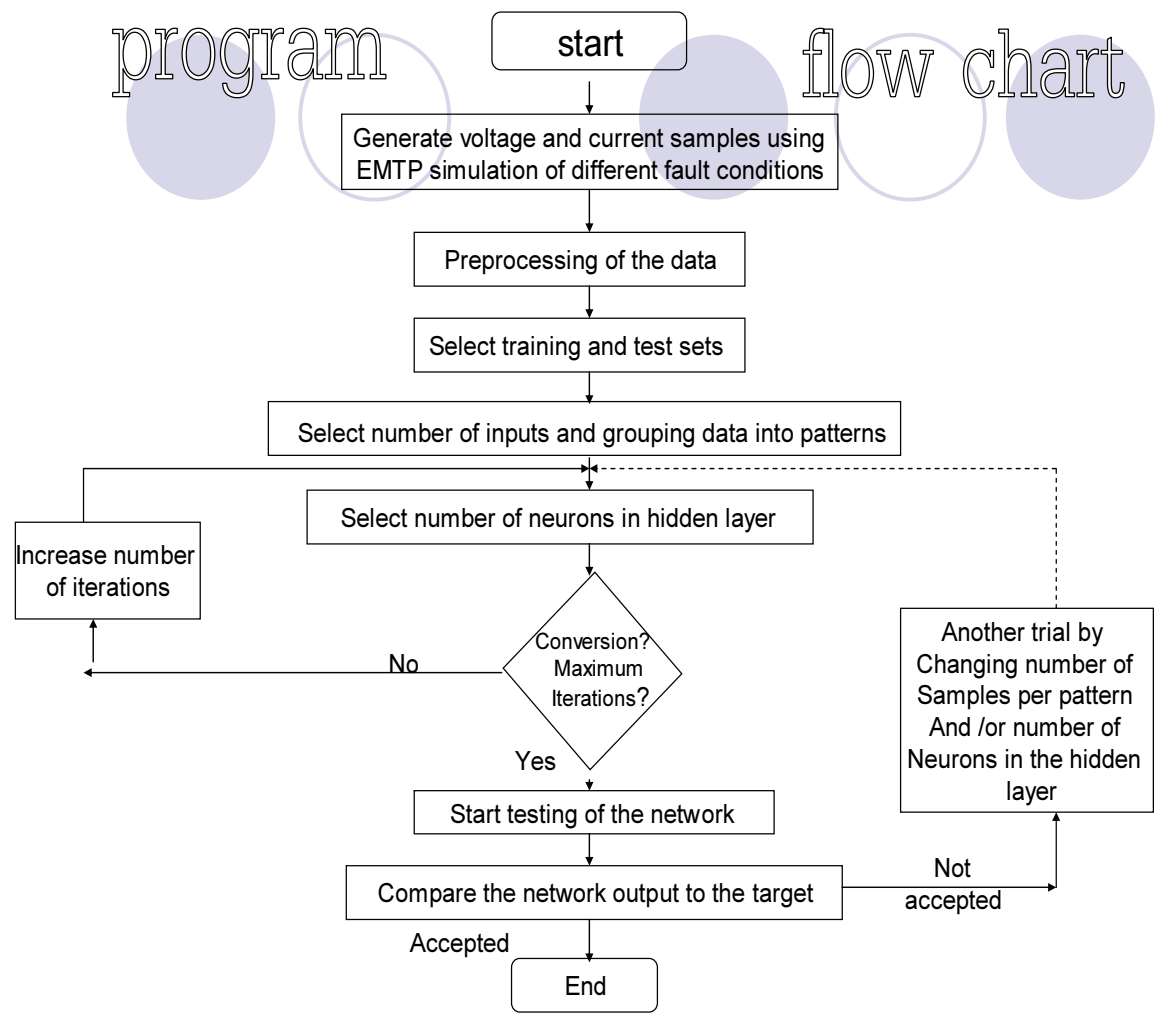

Fig. 3. The ANN design program flow chart.

\section{Sample Training Results}

Figure 4 shows a sample of the training results for ANN1P \& ANN1S, as explained before in the FDS structure. 

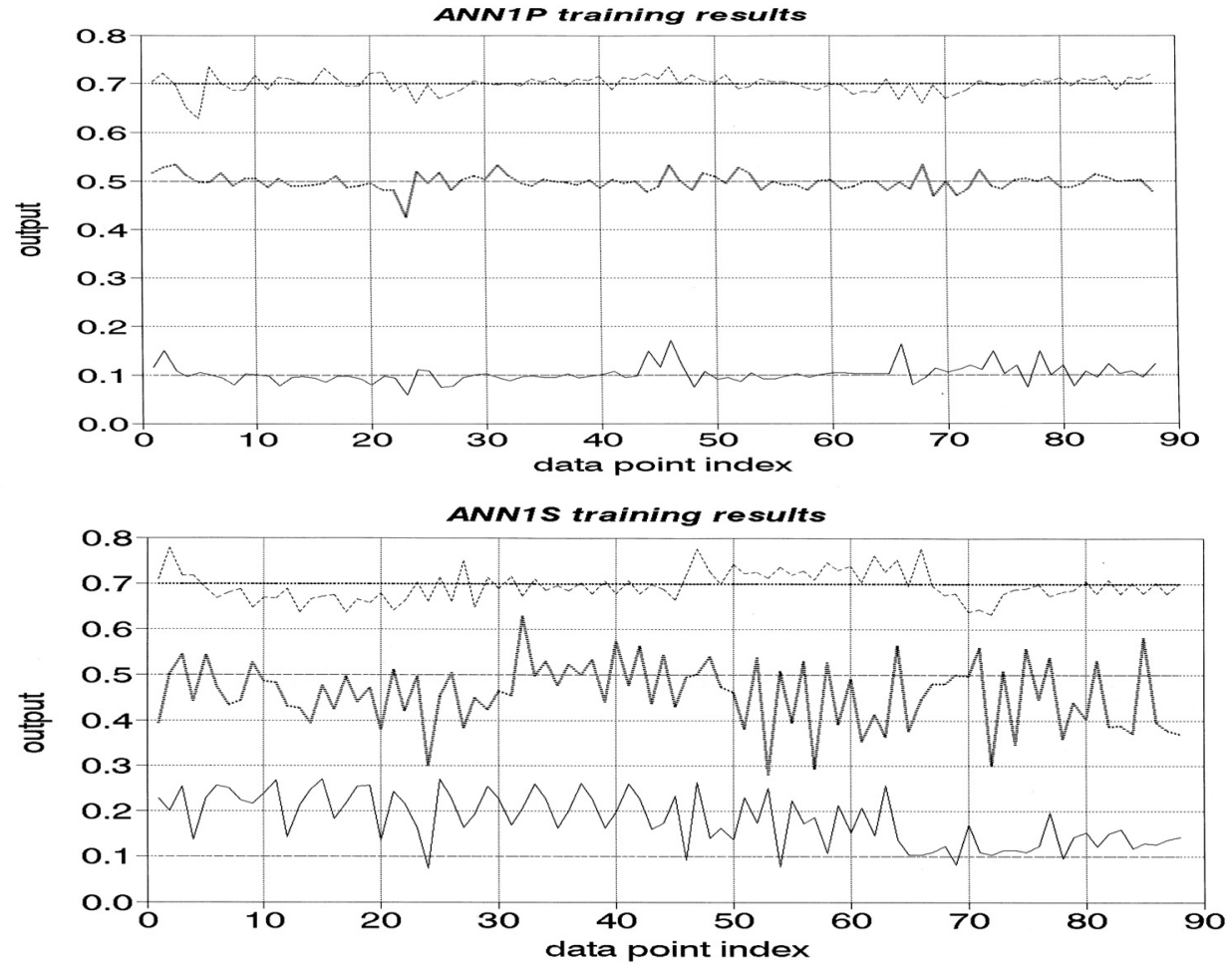

\begin{tabular}{|llllll}
\hline L-G & $\cdots \cdots \cdots \cdots \cdots$ & L-L & L-L-G \\
\hline
\end{tabular}

Fig. 4. Sample training results for ANN1P \& ANN1S. 


\section{بناء نظام لتشخيص الأعطال لمحو لات القوى الكهربائية}

السيد عبد العليم محمد+ ، المعتز يوسف عبدالعزيز، وأمل سيا مصطقى*

قسم هندسة القوى الكهربائية- جامعة عين شمس- القاهرة - مصر،

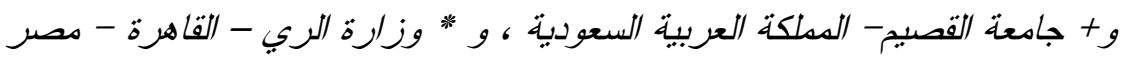

المستخلص . يتتاول هذا البحث بنـــاء نظــام لتـشخيص الأعطــال لمحو لات القوى الكهربائية باستخدام الثبكات العصبية الاصطناعية.

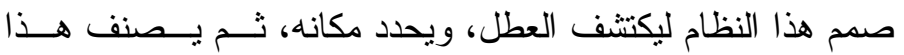

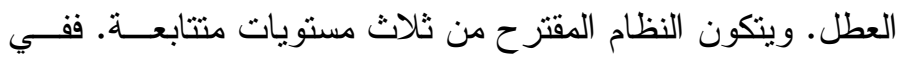
المستوى الأول تتم عملية تجهيز البيانات، وفي المستوى الثاني يتم تصميم شبكة عصبية بغرض اكتشاف العطل وتحديد جانب المحول

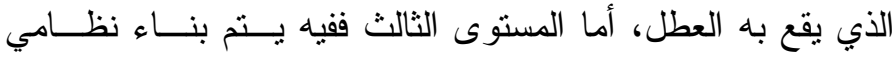

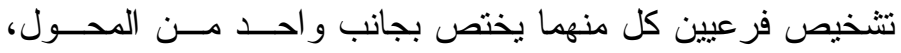

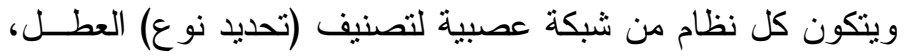

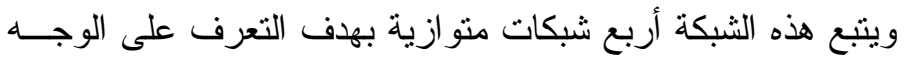

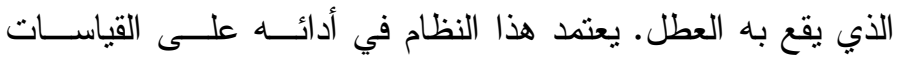

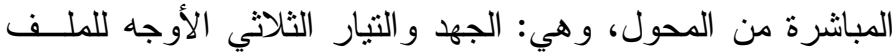
الابتدائي، بالإضافة إلى التيار الثثلاثي للملف الثانوي. تم اســـتخدام

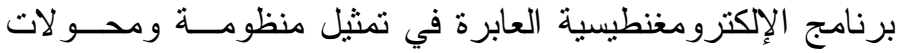

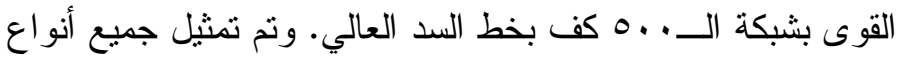

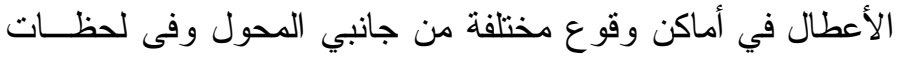

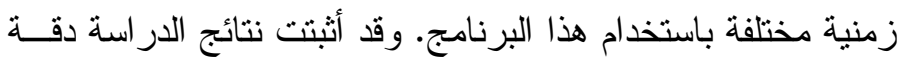
وكفاءة أداء نظام التثخيص المقترح. 